\title{
Manifestation of Color Confinement in the YY Model for Atomic Nuclei
}

\section{-With Implication to Colors, Anti-Colors and Interactions}

\author{
Hongguang Yang, Weidong Yang \\ Munich, Germany \\ Email: hgyang2013@gmail.com
}

How to cite this paper: Yang, H.G. and Yang, W.D. (2020) Manifestation of Color Confinement in the YY Model for Atomic Nuclei. Journal of Modern Physics, 11, 1999-2010.

https://doi.org/10.4236/jmp.2020.1112127

Received: October 29, 2020

Accepted: December 22, 2020

Published: December 25, 2020

Copyright $\odot 2020$ by author(s) and Scientific Research Publishing Inc. This work is licensed under the Creative Commons Attribution International License (CC BY 4.0).

http://creativecommons.org/licenses/by/4.0/

\begin{abstract}
In this paper, a manifestation of the well-known color confinement from the QCD (quantum chromodynamics) in the newly developed YY model for the atomic nucleus is presented. There is a wonderful correspondence between the structural requirements from the YY model and some elementary properties of the color dynamics from QCD. The open questions in the YY model, namely the holding forces for triple nodes and for pairing space links, are exactly covered by the three-color compensation or by the paired color anti-color balance. We will see what colors and anti-colors do mean in the YY model, how up quarks and down quarks get assigned a color or anti-color. We will discover some relationships between gluon-based interactions as described in the standard model and pairing space links in the YY model.
\end{abstract}

\section{Keywords}

Quantum Chromodynamics QCD, Color Confinement, YY Model for Atomic Nucleus, Pairing Space Link PSL, Triple Space Link TSL, Colored up Quark, Colored Down Quark, Colored Hydrogen Nucleus, Colored Helium Nucleus, Colored Helium Isotope Nucleus, Color Confinement Aggregate State CCAS, Color-Balanced PSL, Tumbling of Colored PSLs

\section{Introduction}

The YY model (Ref. [1] and [2]) was first introduced several months ago. The most important subatomic particles (up quark, down quark, neutron and proton) and some complex nuclei (deuterium, tritium and helium) have been described in their structural (spatial) constitutions. A basic understanding of the YY model with its schematic description approach is required to follow the color 
dynamic interpretations here. The content of this paper is a further step in the development of the YY model considering the knowledge artifacts from the standard model and its extension approaches (Ref. [3] [4] [5] [6] [7]).

An important result of the current paper (Sections 2, 3, 4, 5 and 6) will be the assignment of quantum colors to the structural constructs of the YY model, in particular to the up and down quarks, giving an interpretation of the color confinement of the QCD for selected atomic nuclei, from a neutron and a proton, to the complex helium nucleus. The mechanism is applicable to all nuclei described by the YY model.

The other results of the current paper (Section 7) will be the interpretation of the interaction mechanism by color anti-color pairs, which cause the color exchange of two building quarks of an atomic nucleus. Again, the YY model will provide a more detailed plausible visualization of the color anti-color pairs and how they mediate color exchanges. There is a close analogy to the gluon-based interactions.

Furthermore, a very interesting fact will be worked out: The structural make-up of an atomic nucleus can assume a number of defined states, the so-called "Color Confined Aggregate State CCAS" (Section 6). Each CCAS is well described. The set of all CCASs for a certain nucleus can act as a kind of "quantum aggregate". Considerations are made about the relationship to gluon interactions and quantum nature (Section 8).

\section{Assignment of Colors to PSL and Quarks}

The YY model introduced the "Pairing Space Link (PSL)" for building the spatial structure of each atomic nucleus. A PSL consists of a Yang and a Yin, which are marked with "+" and "-" respectively. If we assign a color to them, e.g. "red", we get a colored PSL in Figure 1.
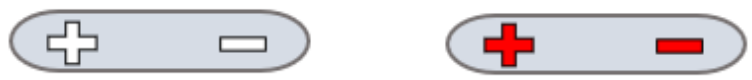

Figure 1. Pairing space link and its coloring in red.

As will be seen later, it is reasonable to consider the Yang end as red and the Yin end as anti-red. This is an important aspect of the YY model for the color manifestation, it associates three colors (red, green and blue) with the positive pool (Yang) and their anti-colors consequently with negative pool (Yin), without using cyan, magenta and yellow.

The YY model described the up quark as an energetic and materialized state combination of two Yang's and a PSL, whereas a down quark is simply an energetic and materialized Yin (Figure 2, left parts). If an up quark is colored green, this means that the PSL is green and the head is white (green, blue and red). To say that a down quark is colored yellow simply means to color its Yin through the yellow (Figure 2, middle and equivalent right-hand side). 

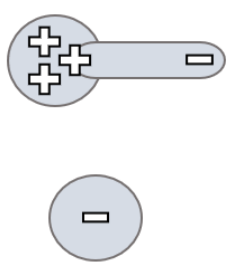
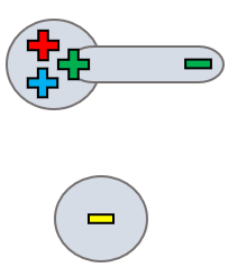

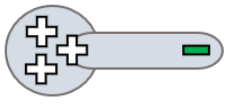

=ニ>

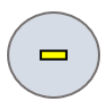

Figure 2. Up quark and down quark (left) and their coloring in green and yellow.

As will be seen later, it is reasonable to consider the color of this up quark simply as green, without necessarily distinguishing between green and anti-green. Similarly, it is reasonable to consider the color of this down quark as yellow, without necessarily distinguishing between yellow and anti-yellow.

\section{Color Confinement within Simple Hadrons}

Based on the structural description of a neutron and a proton in the YY-model (Figure 3, left parts) and based on the color assignments to the quarks in the last section, it is easy to understand the color confinement within a neutron and a proton nucleus (Figure 3, right parts).

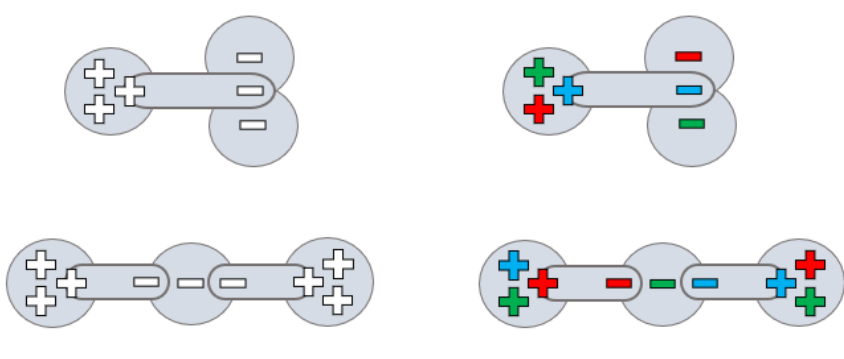

Figure 3. Neutron and proton, their coloring to a confined state.

This current coloration can be considered as an enclosed state: The neutron as an aggregate has assumed a confined state with a blue PSL. Similarly, the proton, as an aggregate, has assumed a confined state with the red and blue PSLs. The current color of each PSL can be understood as a spontaneous state which is "fixed by an observation": For the neutron the PSL color can also take on red or green. For the proton, the colors of two PSLs can take on red-green or blue-green.

\section{Manifestation of Colors, Anti-Colors and Color Confinement in YY Model}

In summary, the colors and anti-colors manifest themselves in (or are associated with) the constituting parts Yang and Yin of the PSL, Figure 4.

The difference between a color and an anti-color is just the different assignments: The color is assigned to yang and the anti-color is assigned to yin. The three PSLs in Figure 4 are called "color-balanced" or "color-symmetric", because the yang and its counter-part yin have the same color. As will be seen (Section 7), interactions will make use of "unbalanced" PSLs, for example the color pair green anti-red. 


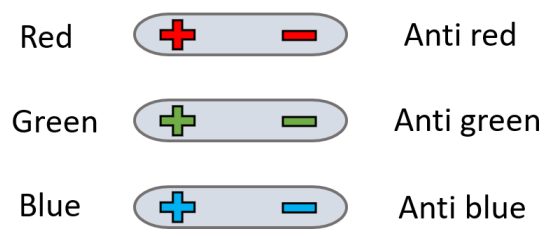

Figure 4. PSL colored in red - anti red, green - anti green or blue - anti blue.

The color confinement in the YY model states that each triple node of yang (or of yin) must be tricolor in red, green and blue (or anti-red, anti-green and anti-blue) to yield white, electrically positively (or negatively) charged, Figure 5.

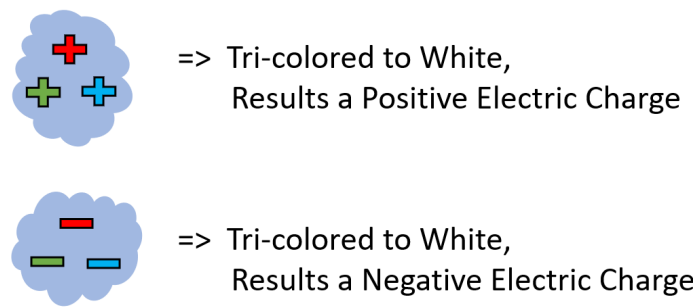

Figure 5. Triple color-charged nodes, resulting a positive or negative electrical charge.

\section{Assignment of Colors to TSL}

The YY model introduced also the "Triple Space Link (TSL)" for constructing complex atomic nuclei. Conceptually, this is a combination of three pairing space links, PSLs. Physically, however, a TSL with triple yang's on the central node (Figure 6, upper left) has a much stronger binding than a TSL with triple yin's on the central node (Figure 6, lower left). The colored TSLs result in Figure 6 on the right, considering the color confinement (red - green - blue yield white in the center).
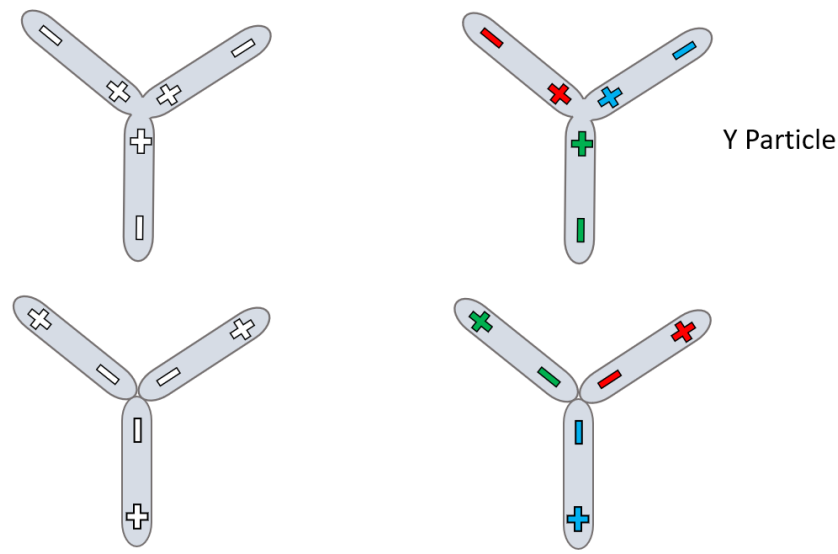

Figure 6. Triple space links, positive and negative centered, and their coloring.

Although the research focus here is on color handling, it should be mentioned that a triple space link with a yang node corresponds to a $\mathrm{Y}$ particle, which is predicted by the YY model, stable and not necessarily elementary, upper right part of 
Figure 6. All nuclear transmutations described by the YY model reserve the triple yang nodes, while triple yin nodes are recombined with quarks and other TSLs, see Figure 7: The shaded parts are all possible combinations on yin nodes.
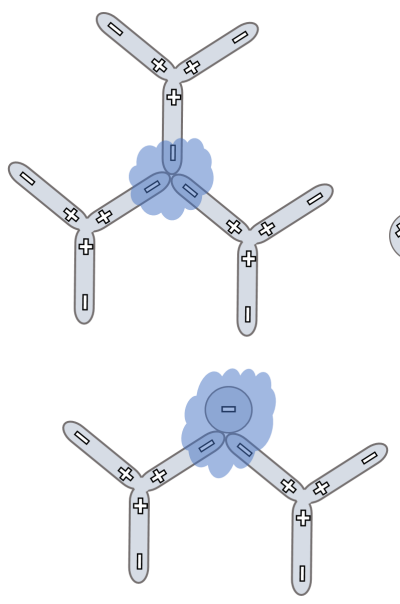
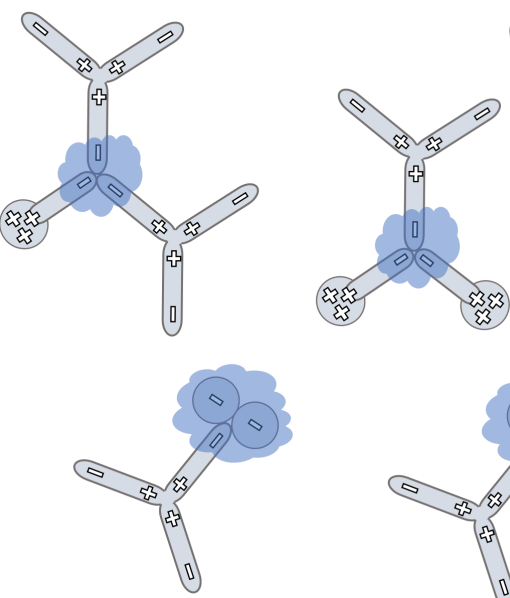
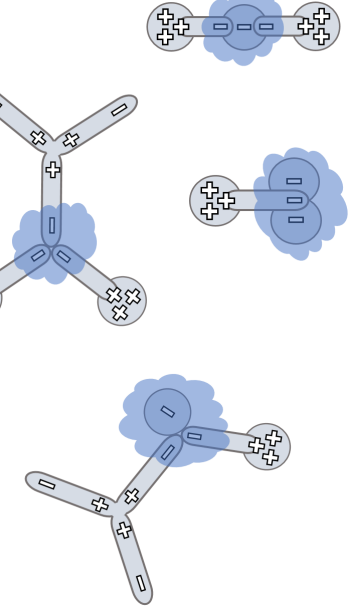

Figure 7. All possible combinations for TSLs with yin nodes.

Based on these color assignments, the YY model is able to balance each complex atomic nucleus to white through colors and anti-colors and to form hadron aggregates.

\section{Color Confinement within Complex Atomic Kernels}

The YY model describes a complex atomic nucleus, e.g. the main proton isotope $\mathrm{H}^{2}$ (deuterium), by using TSL to combine its neutronhead (upper part with two down quarks), protonhead (lower right part with one down quark) and protonids (left and right parts, all up quarks), following the construction rules defined in the YY model (e.g. Internal Charge Balance ICB), Figure 8.

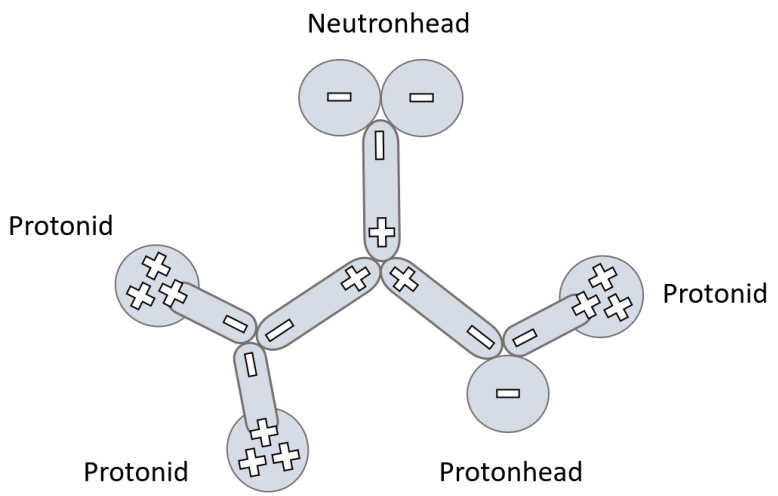

Figure 8. YY model description for atomic nucleus of deuterium.

By coloring all constructing parts (yang's, yin's and consequently PSLs and TSLs), by forcing tri-color compensation at each node (red green blue to white or ant-red anti-green anti-blue to white) and by forcing pairing color balancing 
(red anti-red, green anti-green or blue anti-blue), many special color aggregates may be derived. Figure 9 shows three examples of them.

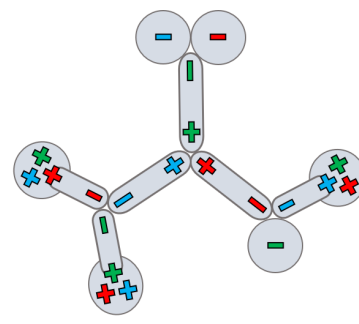

CCAS I

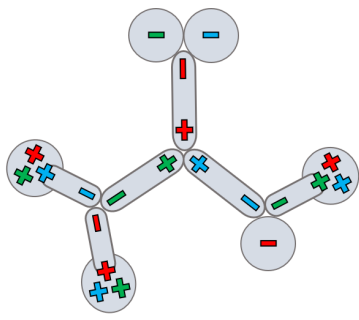

CCAS II

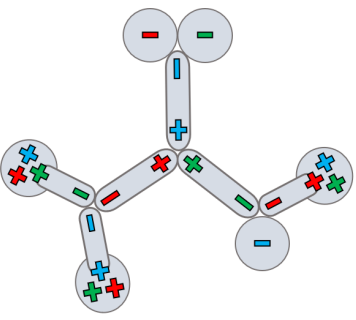

CCAS III

Figure 9. Three samples of color confined aggregate states (CCAS) for a deuterium nucleus.

They are special because compensating all node colors will result the entire aggregate white. We call this combination "Color Confined Aggregate States CCAS". Obviously, there are more such states beyond that showed in Figure 9. It is easy to obtain them by recombining the colors and by following the confinement requirement.

The introduction of the term CCAS is important in considering each atomic nucleus as a conceptual aggregate of a series of slightly different varied color distributions. At this stage of understanding, any CCAS can be considered as a state fixed by observing a quantum system that is determined by the uncertainty: One knows the three possible colors (red, green und blue) for a given PSL, but a certain color (e.g., green) is associated with a fixed state.

It must be possible to map the described coloring behavior of structures and states to the solution space resulting from the theories of symmetric and asymmetric groups, as well as of quantum fields. Further investigations of CCAS and its relation to the standard model are necessary.

Following the descriptions, more complex atomic kernels (tritium, helium and one of its isotope) are given for just one of their all possible CCASs.

Firstly, the proton isotope $\mathrm{H}^{3}$ (tritium) consists of one proton (one protonhead) and two neutrons (two neutronheads) as shown in Figure 10.

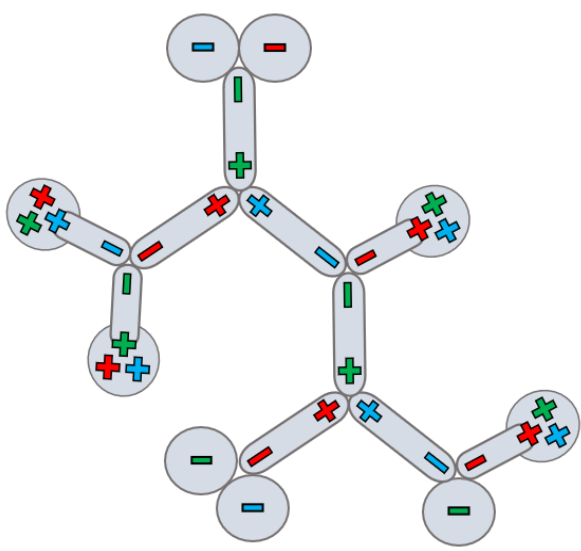

Figure 10. A color confined aggregate state (CCAS) for a tritium nucleus. 
For the next, the helium $\mathrm{He}^{4}$ consists of two protons (two protonheads) and two neutrons (two neutronheads), Figure 11.

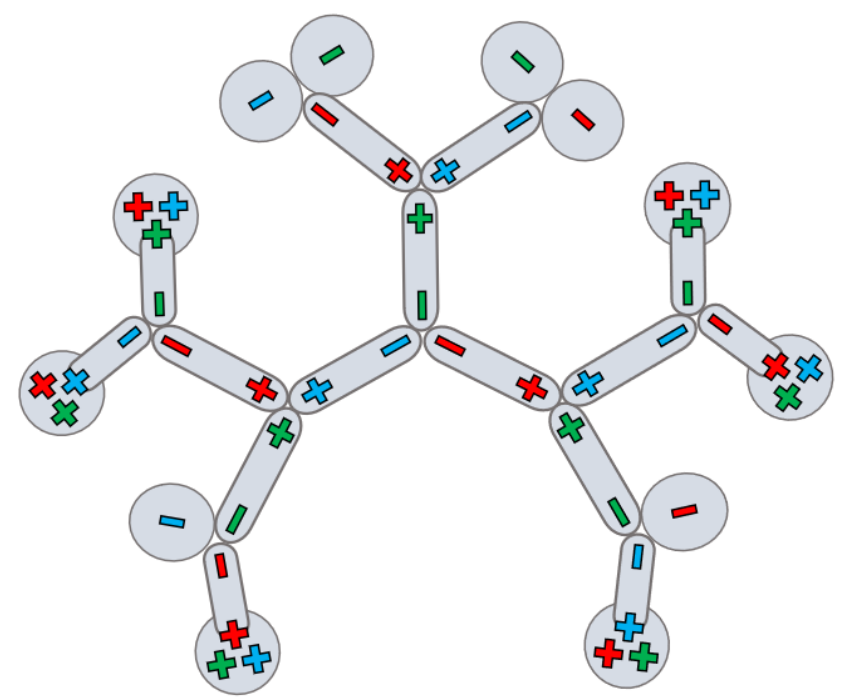

Figure 11. A color confined aggregate state (CCAS) for a helium nucleus.

Finally, the helium isotope $\mathrm{He}^{3}$ consists of two protons (two protonheads) and one neutron (one neutronhead) as shown in Figure 12.

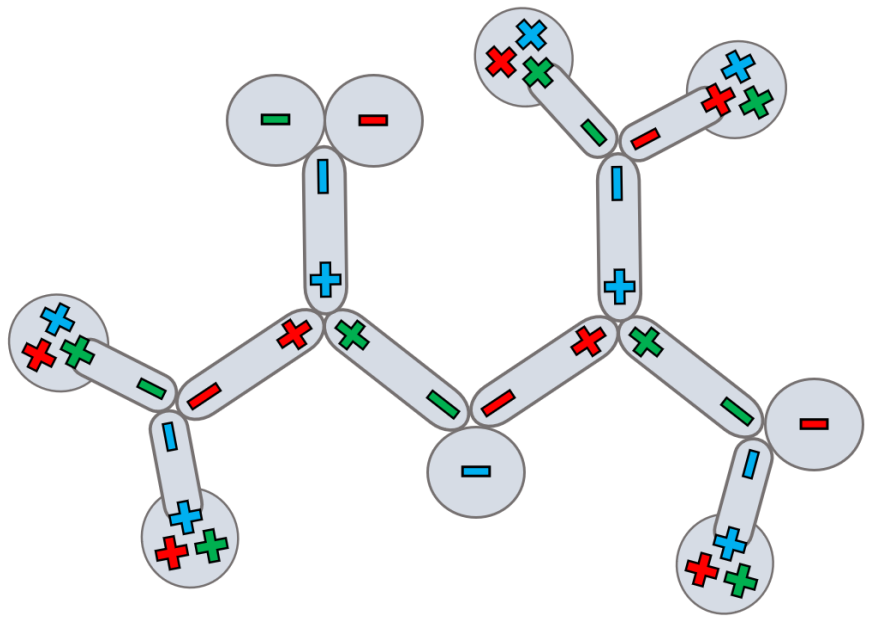

Figure 12. A Color confined aggregate state (CCAS) for a helium isotope nucleus.

\section{Interaction Mechanism by Colored PSLs}

All states described before are "color confined" because all triple nodes (TSLs) are white and all paring space links (PSLs) are balanced. Now the aspect of interaction is considered: How the color of a quark is transmitted to another quark, i.e. the both quarks exchange their colors - this is illustrated by using the example of the deuterium nucleus.

Figure 13 illustrates a change of state from "CCAS I" (color confined) to a new "Not Confined State A": The upper right down quark (anti-red) and the 
linked PSL (anti-green) exchange their colors, so that this down quark becomes anti-green and the PSL receives the anti-red and is now charged to a pair of green anti-red.

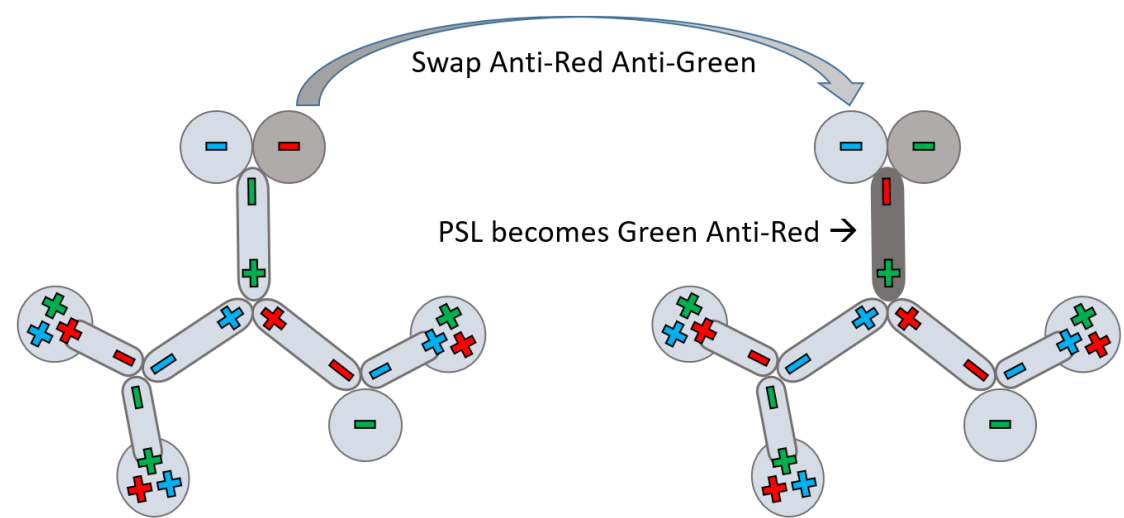

Color Confined Aggregate State I

Not Confined State A

Figure 13. Change of state by swapping the colors of a down quark and the linked PSL.

This participating PSL is now "unbalanced" because it contains two different colors. Therefore, the whole aggregate is called "not confined".

The next change of state is from "Not Confined State A" to "Not Confined State B", Figure 14: The unbalanced PSL (green ant-red) forces itself to balance by pulling the red color at the triple node and giving its green to the adjacent PSL, which loses its red.

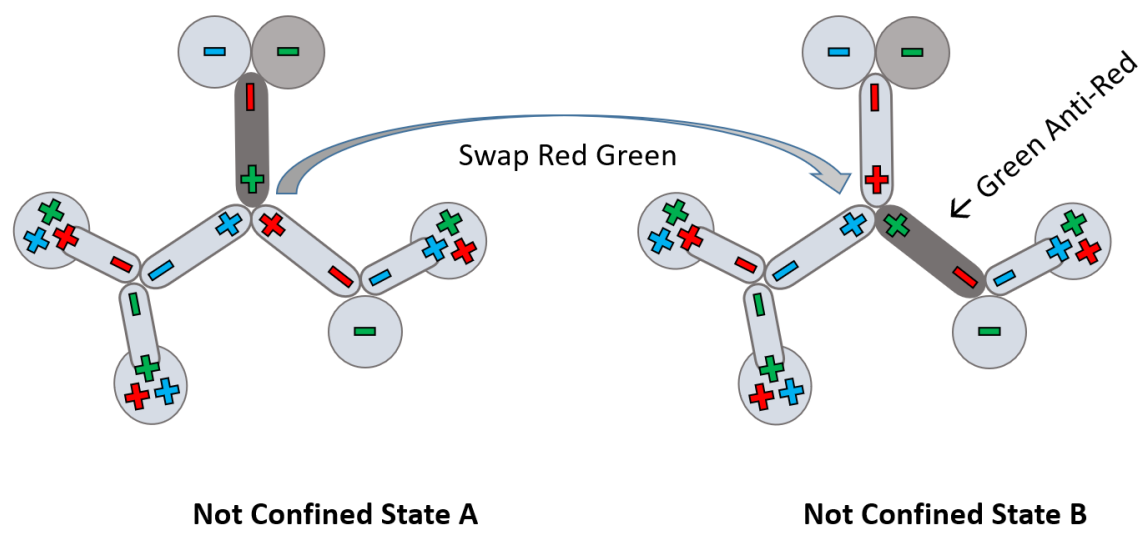

Figure 14. Change of state by swapping the colors of green and red of two linked PSLs.

The first unbalanced PSL gets its color re-balanced and changed its color from original green to current red, whereas the second balanced PSL now becomes unbalanced (green anti-red). It is equivalent to a tumbling movement of the pair green anti-red alongside the PSL chain.

The further change of state from "Not Confined State B" to "CCAS II" (color confined) is done by swapping the anti-red of the unbalanced PSL and the an- 
ti-green of the down quark on the low part, Figure 15.

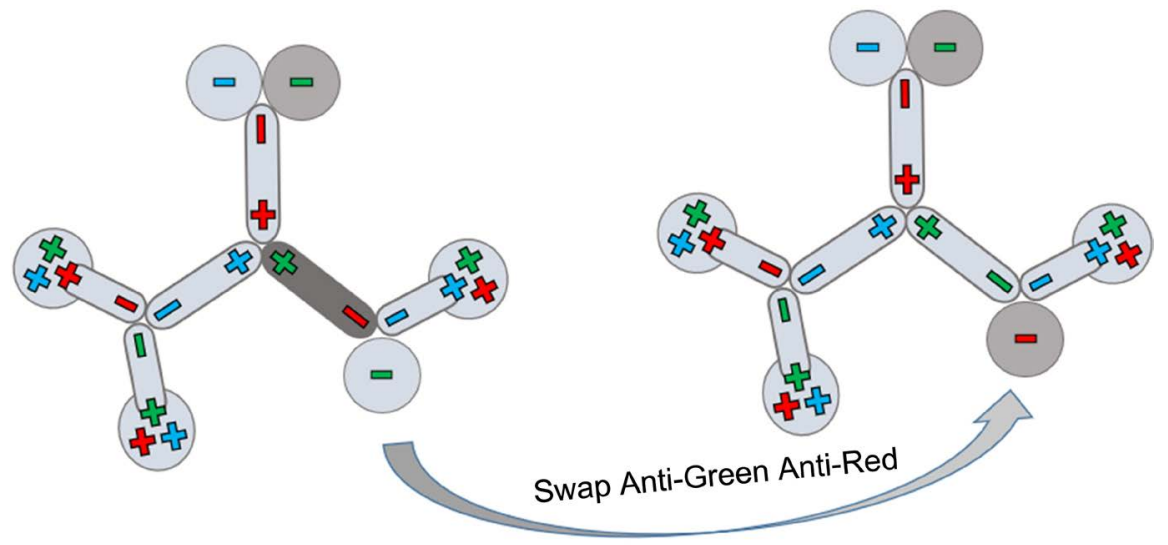

Not Confined State B

Color Confined Aggregate State II

Figure 15. Change of state by swapping the colors of anti-green of down quark and anti-red of PSL.

The end effect of all these interactions is the color exchange of two down quarks, from the original anti-red anti-green to anti-green anti-red. All interactions are submitted by the green anti-red PSL, which runs along the structural chain by "tumbling". The entire aggregate changed its state from "CCAS I" to "CCAS II", with both color state confined.

If we consider only the relay effect of the color and the anti-color, the ending quark of color changes is clearly determined by the three following factors:

$>$ Color of the starting up quark or Anti-color of the starting down quark;

Anti-color of the first PSL;

$>$ The current CCAS, will determine the travel path and the end quark.

In addition, the color exchanges of the two down quarks are "path-symmetric": The change of state from "CCAS II" to "CCAS I" is transmitted by the same pair of green anti-red and follows the same travel path (backwards). Also note that all participating PSLs on the path before and after the change are alternately green and anti-red. This means that the pair of remotely exchanging quarks "will" follow a path with alternating colors of their own.

The previously shown case was the (anti-) color exchange of two down quarks. By the same mechanism two up quarks exchange their colors. The color of an up quark and the (anti-) color of a down quark can also be exchanged with each other and vice versa.

At the end of this section, Figure 16 shows another change of states (CCAS X to CCAS Y) by exchanging the colors of two up quarks, without drawing "not confined" intermediate states.

In Figure 16, the neutronhead (upper node) and one protonid (low node) that do not participate in the exchange are marked in white - also due to the three compensating colors red, green and blue, respectively anti-red, anti-green and anti-blue. 


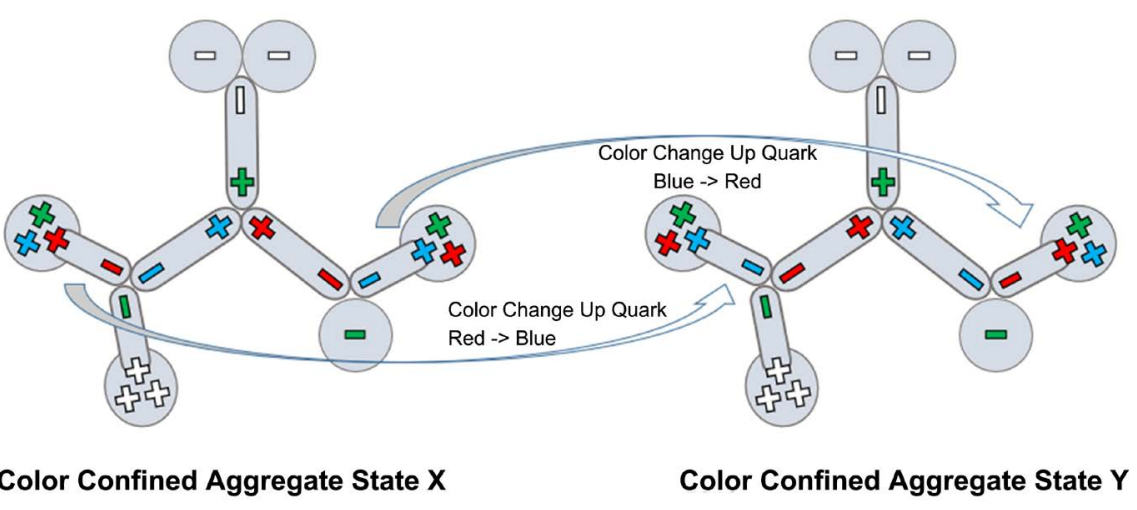

Figure 16. State change by swapping colors of two up quarks (Red $\leftarrow \rightarrow$ Blue).

\section{Further Considerations}

The manifestation of the color confinement from the standard model is closely related to the color assignment to the structural constructs (yang and yin) from the YY model. "Confinement" in the sense of the YY model means that each positively charged node is colored by red, green, blue, whereas each negatively charged node is colored by anti-red, anti-green and anti-blue. A color is always assigned to a yang node, whereas an anti-color is always assigned to a yin node. The "confinement" in the YY model also requires that each PSL must be color balanced (either red anti-red or green anti-green or blue anti-blue).

The color exchange of the quarks takes place via the interaction mechanism described above. It is transmitted by the "unbalanced PSLs" as an asymmetric color and anti-color pair. It is therefore very similar to the interactions transmitted by gluons, which form the basis of the strong forces in the standard model. There is much potential for studying the internal relationships between the gluons and the PSLs. For example, it is conceptually simple to represent the unbalanced PSLs in Figures 13-15 as gluon tubes (Ref [8] and [9]). All this is logical, and the question is justified how this color exchange should be performed statically or dynamically.

The cause of the initial color change is unclear (for example, the change of state from "CCAS I" to "Not Confined State A" in Figure 13). Possibly there is some kind of "ghost" inside or around the aggregate that causes the color change. It is also unclear whether the color change of several quark pairs occurs simultaneously and whether and how the tumbling PSLs interfere with each other, especially on the TSL node. In view of the color changes described by the standard model, the two similar questions arising from this are also not yet answered properly.

There are two possible ways to gain a better understanding, which can lead to achieve interesting results through more in-depth research.

One is based on the idea that the strong forces that hold the surrounding quarks of an atomic nuclear aggregate interact statically (TSL nodes, electrically positive and negative alternating, plus PSL links, color and anti-color balanced). The nucleus must always be a CCAS. The detailed color change mechanism as a 
thinking aid of the standard model becomes imaginary. This consideration requires a multiple solution of the quantum field potential for the entire aggregate-think of a flock of birds finding its flight formation interactive and self-organized.

The other way is the "vertical" superposition of the multiple color states by considering them as quantum possibilities in a broader sense the Schrödinger cat analogy: In the whole kernel aggregate, the colors of the PSLs change within the possible values, forced by the "color rotations" of the adjacent TSLs. The symmetry between the color confined aggregate states is broken locally by an observation that provides an immediate snapshot. This way is more compatible to the usual interpretation of interactions for strong forces.

Finally, we mention the main difference between the methodological approach of the YY model and other schematic approaches to nuclear models, such as preon model (Ref [10]) or molecular nucleon model (Ref [11]). The YY model uses well-known basic artifacts of the standard model to build an architecture that allows to include more and more facts in a clear and consistent way. Regarding the color considerations of this paper, which are based on the model construction of the last paper, the authors believe that the goal of this approach has been achieved.

\section{Conflicts of Interest}

The authors declare no conflicts of interest regarding the publication of this paper.

\section{References}

[1] Yang, H. and Yang, W. (2020) Extending Standard Atomic Kernel Model with New Interpretation of Strong Forces. https://arxiv.org/abs/2006.15695

[2] Yang, H. and Yang, W. (2020) A Generic Description Model for the Structure of Atomic Nucleus with New Interpretation of the Strong Forces. Journal of Modern Physics, 11, 1132-1156. https://doi.org/10.4236/jmp.2020.118071

[3] Di Giacomo, A. (2006) Confinement of Color: Open Problems and Perspectives. AIP Conference Proceedings, 842, 216. https://arxiv.org/abs/hep-lat/0602011 https://doi.org/10.1063/1.2220230

[4] Di Giacomo, A. (2007) Recent Progress on Color Confinement. AIP Conference Proceedings, 964, 348. https://arxiv.org/abs/0710.1164 https://doi.org/10.1063/1.2823875

[5] Braun, J., Gies, H. and Pawlowski, J.M. (2007) Quark Confinement from Color Confinement. https://arxiv.org/abs/0708.2413

[6] Hansson, J. (2000) A Simple Solution to Color Confinement. https://arxiv.org/abs/hep-ph/0011060

[7] Tornqvist, N.A. (2007) The Scalar Mesons, Symmetry Breaking, Three Colors and Confinement. https://arxiv.org/abs/hep-ph/0606041

[8] Ying, C. (2020) Confinement and the Global SU(3) Color Symmetry. https://arxiv.org/abs/2010.00431

[9] Baker, M., Cea, P., Chelnokov, V., Cosmai, L., Cuteri, F. and Papa, A. (2019) Isolat- 
ing the Confining Color Field in the SU(3) Flux Tube. The European Physical Journal C, 79, Article Number: 478. https://arxiv.org/abs/1810.07133 https://doi.org/10.1140/epjc/s10052-019-6978-y

[10] Dugne, J.J., Fredriksson, S. and Hansson, J. (2002) Preon Trinity: A Schematic Model of Leptons, Quarks and Heavy Vector Bosons. Europhysics Letters, 60, 188. https://doi.org/10.1209/epl/i2002-00337-8

[11] Ho, V.B. (2020) Molecular Structure of Atomic Nucleus. Journal of Modern Physics, 11, 1395-1409. https://doi.org/10.4236/jmp.2020.119087 